\title{
Dynamic Error Characteristics of Touch Trigger Probes Fitted to Coordinate Measuring Machines
}

\author{
Roland P. Johnson, Qingping Yang, Member, IEEE, and Clive Butler
}

\begin{abstract}
This paper discusses the dynamic error characteristics of touch trigger probes used with coordinate measuring machines. During the investigation, a number of important parameters have been identified, including measurement speed, probe longitude, approach distance, probe latitude, stylus length/stylus tip diameter, probe orientation, operating mode (scanning and nonscanning), scan pitch, preload spring force (gauging force), probe type, and the surface approach angle. This paper presents the detailed experimental design and the results obtained from the systematic experiments. These results have led to some useful recommendations for the reduction of the probe dynamic errors. Some of these recommendations included the selection of the optimum measurement speed, the setting of the preload spring force, and the choice of the probe type.
\end{abstract}

Index Terms - Dynamics, error analysis, inspection, measurement, position measurement, transducers.

\section{INTRODUCTION}

$\mathbf{C}$ OORDINATE measuring machines (CMM's) have been increasingly used in industry for measuring the dimensions of a large range of engineering components. Due to the demand for shorter cycle times of the measurement tasks, for example, when scanning a component with a high density of gauging points on the work surface, there is an increased requirement for faster $\mathrm{CMM}$ operation. As a result the influence of the dynamic errors of the CMM system will increase. Since most CMM's are fitted with touch trigger probes and because one of the largest sources of measurement error in the CMM system is the touch trigger probe itself, it is very important to gain an indepth knowledge of the characteristics of the probe dynamic errors so that the dynamic performance of touch trigger probes may be improved, which was the main purpose of the investigation.

There appeared to be little work specifically on the dynamic errors of touch trigger probes although there was general literature on probes and CMM's. Bosch [1] briefly described the dynamic measurement errors generated by the machine. Weekers and Schellekens [2] and $\mathrm{Mu}$ et al. [3] examined the dynamic errors of the CMM system for fast probing, i.e., the combined machine and probe dynamic errors were assessed. Other references on probe error modeling and correction are [4]-[11].

Manuscript received May 18, 1998; revised November 9, 1998.

The authors are with the Brunel Centre for Manufacturing Metrology, Brunel University, Uxbridge, Middlesex, UB8 3PH, U.K. (e-mail: emstqqy@ brunel.ac.uk).

Publisher Item Identifier S 0018-9456(98)09766-6.

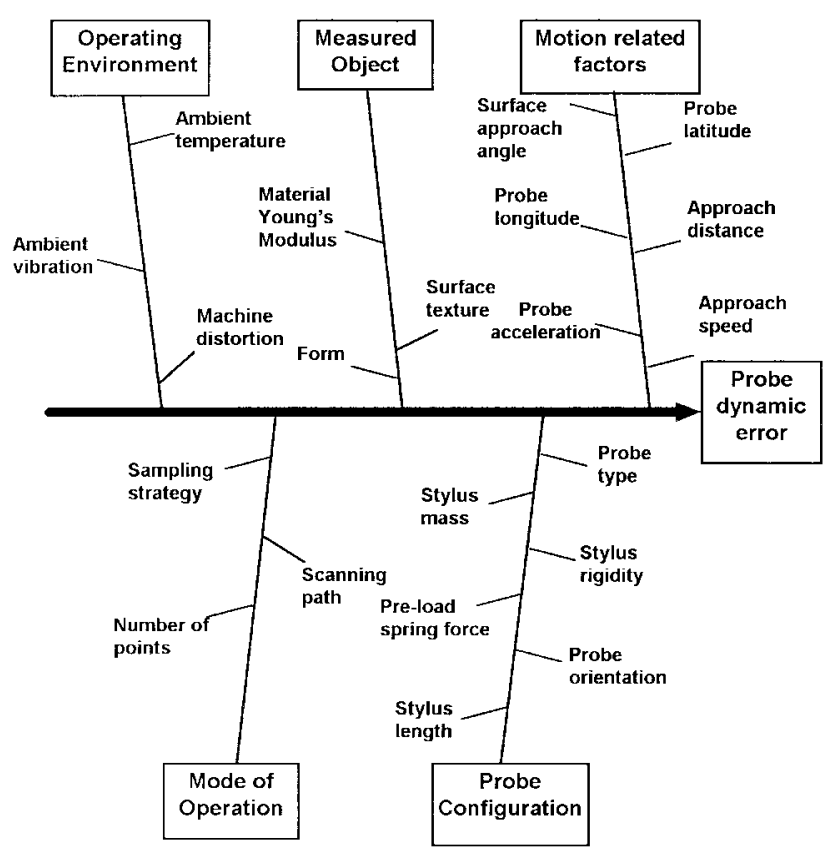

Fig. 1. Fishbone diagram for assessing the probe dynamic measurement error.

\section{Probe DYNAMIC ERRORS}

The probe dynamic measurement errors are influenced by a number of factors, which may be identified and illustrated using a fishbone diagram (Fig. 1).

\section{A. Motion-Related Factors}

When a probe makes contact with the measured surface, the impact force is influenced by several factors including probe approach speed, probe acceleration, approach distance, and probing angles (longitude, latitude).

For example, the higher the probe approach speed, as the stylus tip contacts the work surface, the greater the dynamic forces on the probe system. There would be more flexing and distortion of the stylus/probe assembly, which would increase the measurement error. On the other hand, at very low probe approach speeds, as the probe trigger point is approached, electrical contact noise becomes significant. In practice, the speed is often kept constant so as to minimize the measurement error.

\section{B. Probe Configuration}

On contacting the measured surface, the probe dynamic response of the impact force depends generally upon the probe 


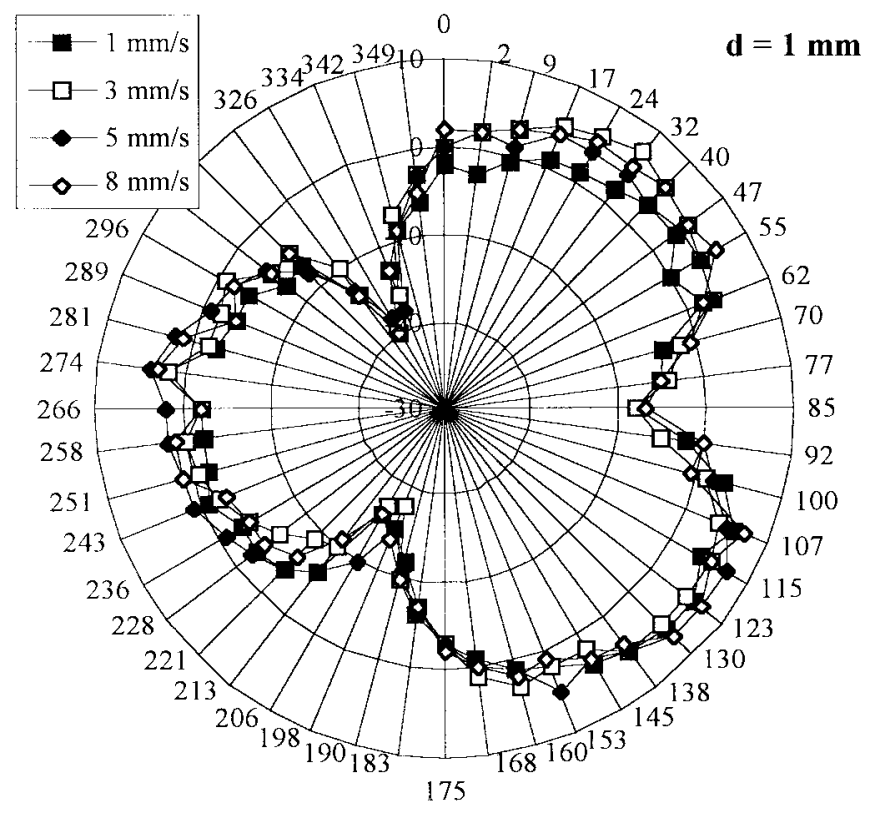

Fig. 2. Actual probe error $(\mu \mathrm{m})$ versus longitude (degrees) (normal preload spring force; approach distance $d=1 \mathrm{~mm}$ ).

and stylus mass and rigidity, in particular, probe preload spring force, probe orientation, and stylus length.

The greater the preload spring force (or gauging force), the greater the contacting force required to activate the probe and thus, the greater the errors would be. The spring force must be high enough to guarantee good kinematic mount contact, but not be so low that false triggering occurs when the probe is traversed from one gauging point to another.

The orientation angle of the probe (e.g., whether vertical or horizontal) would influence the measurement error due to the variations of the gravitational forces.

\section{Measured Object}

The measured object may also influence the probe dynamic performance. For example, if the component is made of soft deformable material, its structure distorts under probe contact.

The probe pretravel may also cause, in addition to the lobing errors, frictional errors due to the possible sliding between the stylus tip and test piece when the probe approaches the surface at nonright angles. This type of error is also influenced by the surface texture (roughness, waviness) of the measured object.

\section{Mode of Operation}

The mode of operation refers to how the measured points are sampled with the probe and is closely related to the measurement sampling strategy. The scanning path of the probe around the work would influence the measurement error since the scanning path would alter factors such as the probe longitude, approach distance, etc. The number of points per gauging surface will also influence the measurement uncertainty, which, in general, decreases with the number of measurement points.

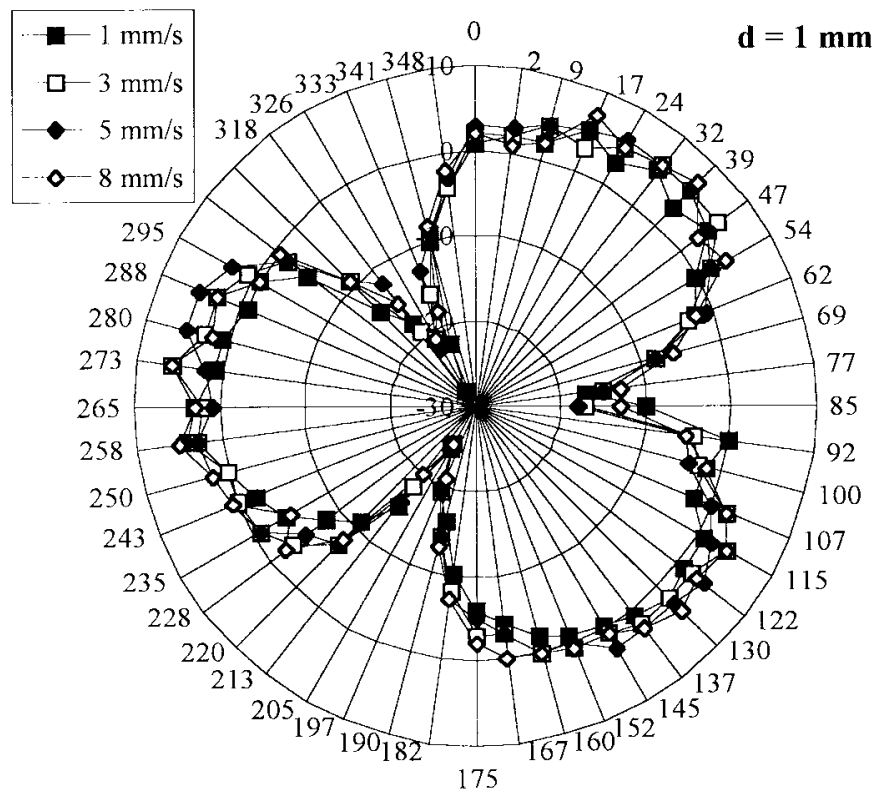

Fig. 3. Actual probe error $(\mu \mathrm{m})$ versus longitude (degrees) (high preload spring force; $d=1 \mathrm{~mm}$ ).

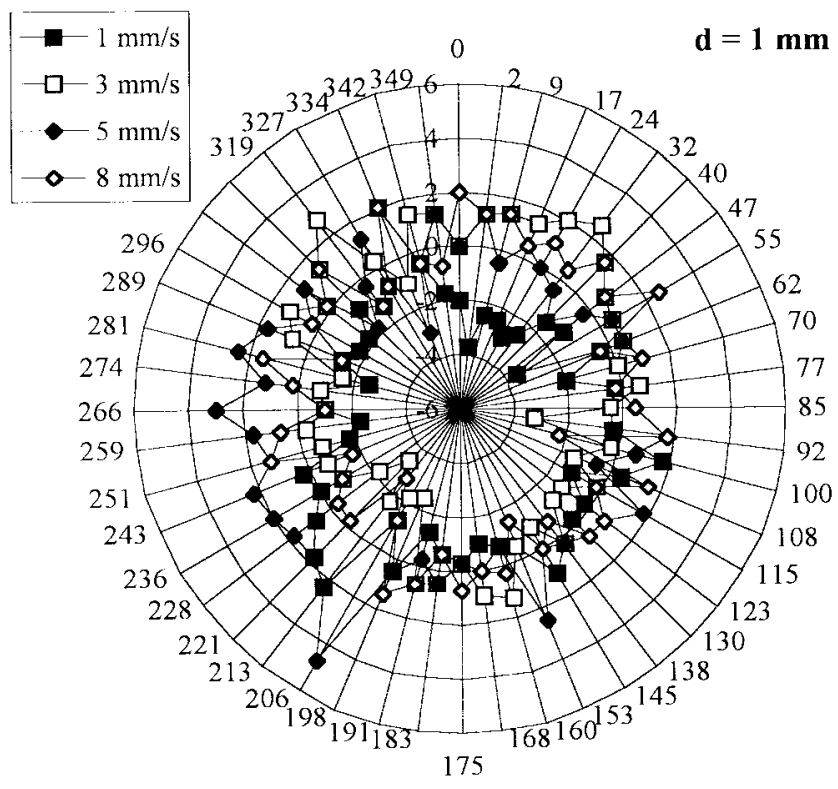

Fig. 4. Dynamic error (micrometers) versus longitude (degrees) (normal preload spring force; $d=1 \mathrm{~mm}$ ).

\section{E. Operating Environment}

Probe dynamic errors may also arise from its operating environment. They can be caused directly by the machine structural distortions due to the mass of the CMM sliding carriages. Some of the static structural errors may already be compensated using computer software (error mapping), but the dynamic errors are usually not. Of course, the computer compensation software may introduce mathematical errors of its own. The ambient vibration, coupled through the machine, may also result in dynamic measurement errors.

Although the thermal drift in a temperature-controlled environment is usually small, large variation in air temperature, 


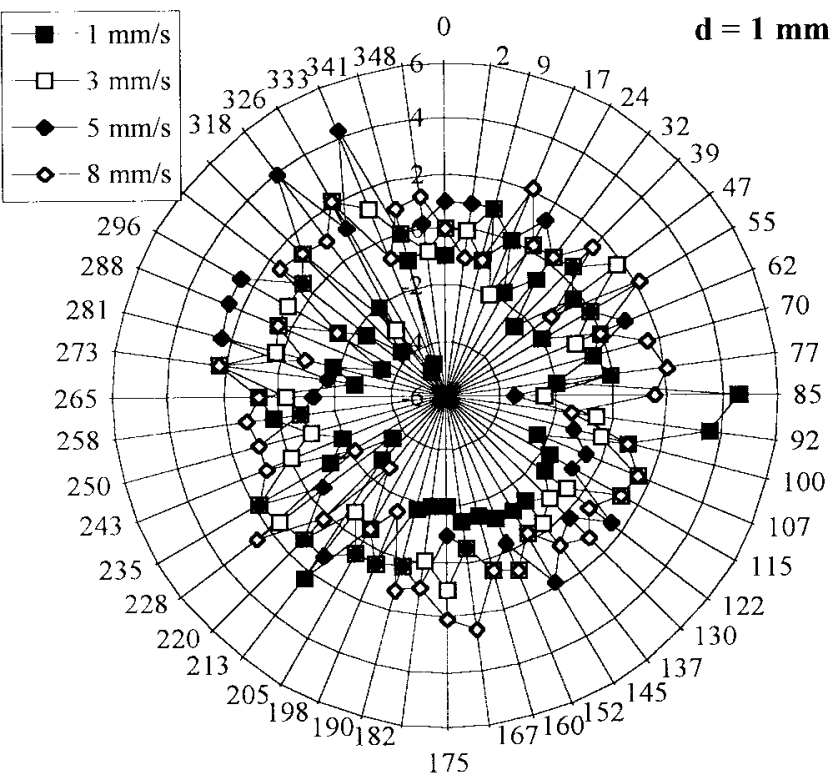

Fig. 5. Dynamic error (micrometers) versus longitude (degrees) (high preload spring force; $d=1 \mathrm{~mm}$ ).

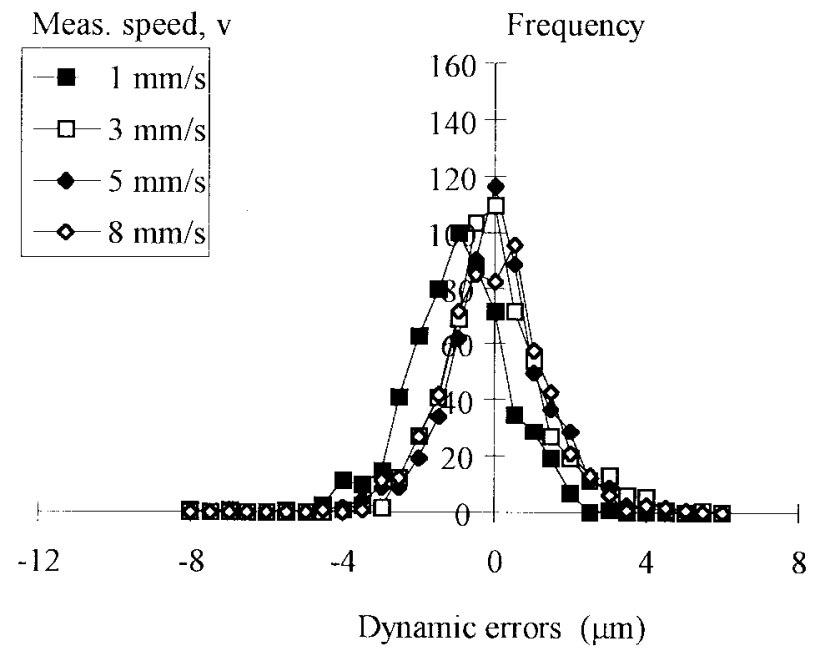

Fig. 6. Histogram of the dynamic error with normal preload spring force.

e.g., on the manufacturing shop floor, would influence the dynamic error characteristics due to thermal expansion of the $\mathrm{CMM} /$ probe system and work piece.

\section{EXPERIMENT}

Since probe dynamic errors are influenced by a number of factors, as described above, it was necessary to select the most important ones for experiment. This was done by means of brainstorming, together with the above considerations. The following test factors were varied at several different levels during the experiments.

The measurement speed, $v$, was varied at levels of 1,3 , 5 , and $8 \mathrm{~mm} / \mathrm{s}$, where 1 and $8 \mathrm{~mm} / \mathrm{s}$ are the lowest and highest speeds possible, respectively; while the recommended measurement speed is $3 \mathrm{~mm} / \mathrm{s}$. The approach distance, $d$, was varied at levels of $0.2,0.4,0.6,0.8,1,2,3,4,5,10,20$, and $30 \mathrm{~mm}$, which covers the smallest to the highest approach

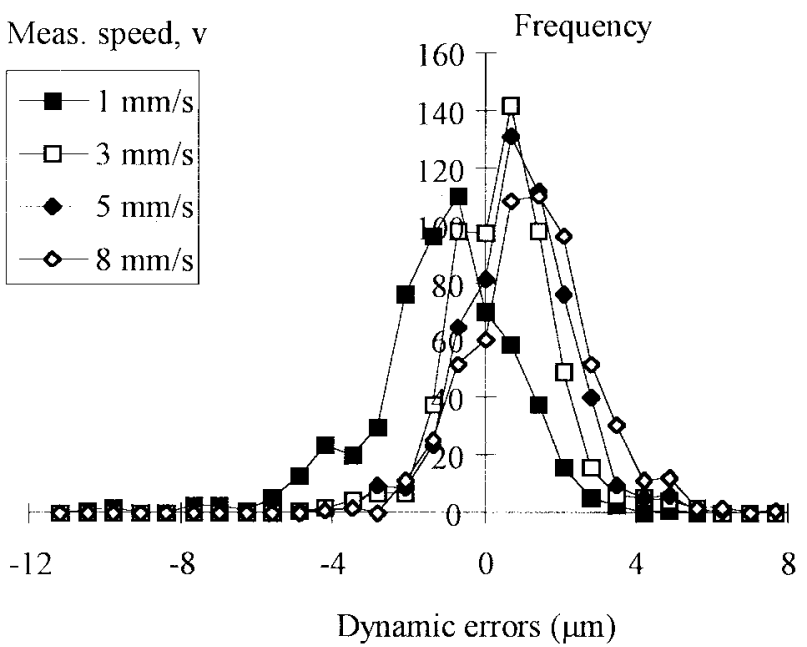

Fig. 7. Histogram of the dynamic error with high preload spring force.

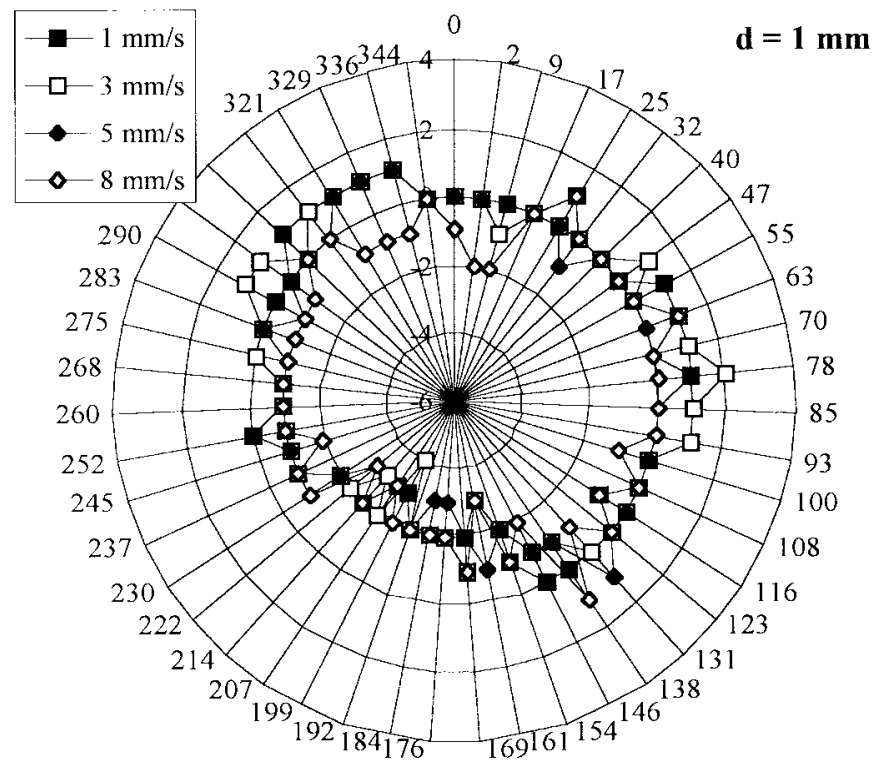

Fig. 8. Actual error $(\mu \mathrm{m})$ versus longitude (degrees) (TP7; $d=1 \mathrm{~mm}$ ).

distances likely to be used in industrial practice. The probe longitude was varied from $0^{\circ}-360^{\circ}$ (with 12 to 48 levels).

Other factors varied in the tests included:

- probe latitude $\left(0^{\circ}\right.$ or $\left.45^{\circ}\right)$;

- stylus length/stylus tip diameter $(30 / 2$ or $80 / 5 \mathrm{~mm})$;

- probe orientation (vertical or $45^{\circ}$ ),

- operating mode (Geopak or Scanpak);

- scan pitch $(80 \%$ or $200 \%)$;

- preload spring force (normal or increased by three turns corresponding to $35 \%$ increase in pretravel);

- probe type (3D TP2-5 way or TP7);

- surface approach angle $\left(70^{\circ}-90^{\circ}\right.$ in $2^{\circ}$ steps).

The experiments were based upon a medium accuracy moving bridge CMM in conjunction with a touch trigger probe (TP2 or TP7). A one-inch calibration master sphere was used as the mechanical reference artifact. The effective stylus tip diameter was calibrated at the beginning of each test run. Whenever the artifact or probe was handled/adjusted, 


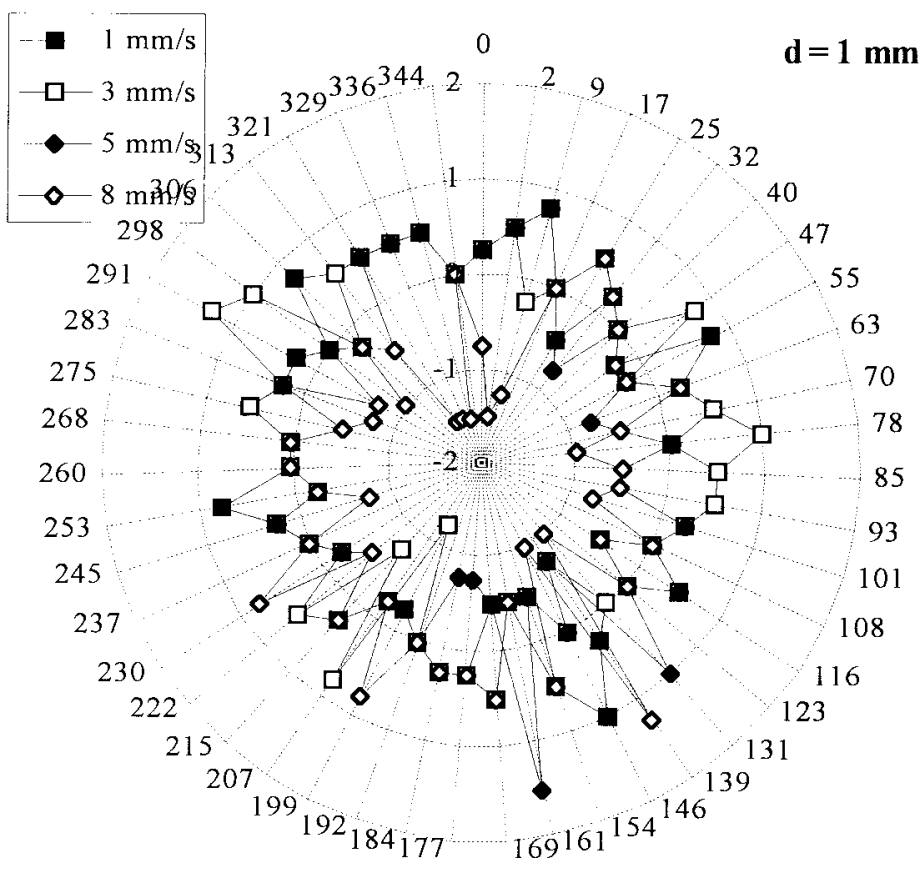

Fig. 9. Dynamic error, $z$ (micrometers) versus longitude (degrees) (TP7; $d=1 \mathrm{~mm}$ ).

a minimum temperature stabilization time of one half hour was allowed in order for the probe/artifact to reach thermal equilibrium. All the tests were conducted in a temperature controlled laboratory at a constant ambient temperature of 21.6 ${ }^{\circ} \mathrm{C} \pm 0.1{ }^{\circ} \mathrm{C}$.

\section{RESULTS AND DISCUSSIONS}

The test configuration for all the results described in this section utilized stylus length $=80 \mathrm{~mm}$, stylus tip diameter $=5 \mathrm{~mm}$, probe orientation $=$ vertical, latitude $=0^{\circ}$ (i.e., equatorial), mode of operation $=$ scanning, and scan pitch $=$ $80 \%$.

For these tests, the measurement error was calculated as the difference between the measured radius (as output directly on the CMM computer) and the actual nominal radius of the master $(12.700 \mathrm{~mm})$. Fig. 2 shows a polar plot of the actual measurement error versus longitude for the normal preload spring force and Fig. 3 for the high spring force configuration.

The above polar plots clearly show the probe lobing characteristic and some random/systematic variation of the actual measurement error with speed. The probe dynamic error may be estimated as the actual probe error minus the mean error over four speeds at a specific longitude. Shown in Fig. 4 is a polar plot of the dynamic errors for the normal preload spring force test and Fig. 5 for the high spring force test.

The above Figs. 4 and 5 show that there was very small variation of the dynamic error with longitude. Figs. 6 and 7 present the histograms of the dynamic errors versus speed for the normal and high preload spring force test, respectively.

Figs. 6 and 7 have confirmed that a higher preload spring force causes larger probe dynamic errors.

The effect of probe type on the dynamic error was also investigated. The actual measurement errors with the TP7

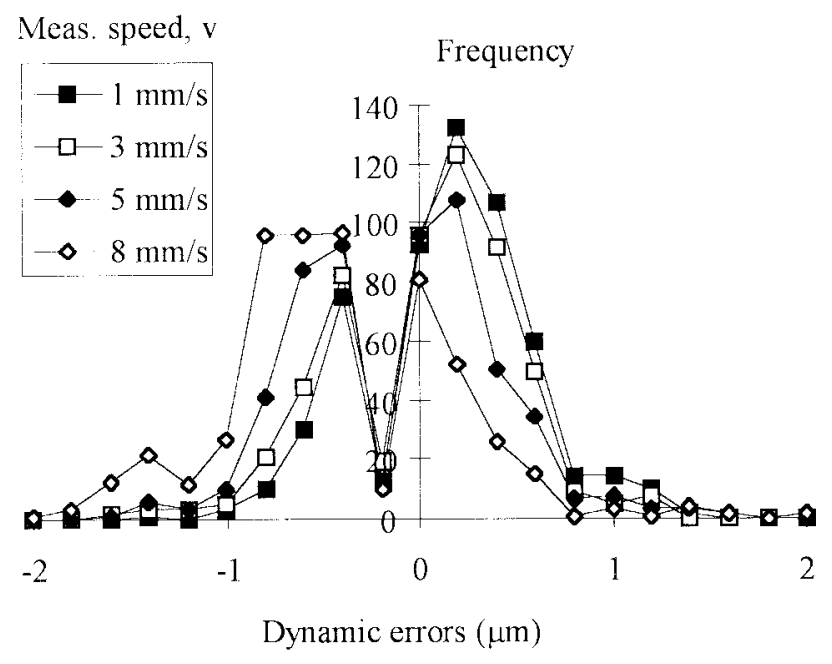

Fig. 10. Histogram of the dynamic errors with the TP7 probe.

probe are shown in Fig. 8, which may be compared with Fig. 2 (TP2 with normal spring force).

Fig. 9 gives the dynamic errors for the TP7 probe. This polar plot may be compared with Fig. 4 (TP2 with normal spring force).

The histogram of the dynamic errors for the TP7 probe is given in Fig. 10, which may be compared with Fig. 6 (TP2, normal spring force).

With the TP7, the absolute variation of measurement error with speed was significantly decreased. However, since the general level of error was also significantly decreased, the effect of speed on the measurement error was actually more dominant if viewed as a fraction of the total error. Note that the frequency distribution in Fig. 10 appears to be bimodal (not Gaussian). 
It can be seen from the experiments that setting the measurement speed to 3 or $5 \mathrm{~mm} / \mathrm{s}$ generally produced the lowest dynamic errors. Also, as the mean dynamic errors changed from the most negative to the most positive values, the speed curves were generally in the order of $1,3,5$, and $8 \mathrm{~mm} / \mathrm{s}$ (except for the TP7 where the speed curve order was reversed, i.e., $8,5,3$, and $1 \mathrm{~mm} / \mathrm{s}$ ).

\section{CONCLUSIONS}

The probe dynamic errors are dependent upon a number of factors. The results of some initial experiments have been presented. The following conclusions can be drawn from the above discussions.

1) Probe gauging force (preload spring force) has a very strong effect on the dynamic measurement error. Its effect on the dynamic error was more significant than the effect of any of the other test control factors. To avoid large errors, it is necessary to set it as low as possible.

2) There exists an optimum measurement speed for dynamic performance. With the $\mathrm{CMM} /$ probe used in the test, the optimum measurement speed range is $3-5 \mathrm{~mm} / \mathrm{s}$.

3) Probe dynamic errors vary significantly according to the type of probe. With the two probe types tested, the TP7 probe gives much better performance.

Further experimental results and discussions will be presented in [11].

\section{ACKNOWLEDGMENT}

The authors wish to thank N. Wells of Renishaw for conveying useful information related to the operation of touch trigger probes. The technical discussions with C. Reeves and M. Kuscher of Mitutoyo are gratefully acknowledged.

\section{REFERENCES}

[1] J. A. Bosch, Ed., Coordinate Measuring Machines and Systems. New York: Marcel-Dekker, 1995.

[2] W. G. Weekers and P. H. J. Schellekens, "Assessment of dynamic errors of CMM's for fast probing," CIRP Ann. Manufact. Technol., vol. 44, no. 1, pp. 469-474, Jan. 1995.

[3] Y. Mu, C. Dong, J. Guo, and G. Zhang, "Dynamic error compensation of coordinate measuring machines for fast probing," in Proc. SPIE-Int. Soc. Opt, Eng., vol. 2899, pp. 6-11, Sept. 1996.

[4] C. Butler, "Investigation into the performance of probes on coordinate measuring machines," Ind. Metrol., vol. 2, no. 1, pp. 59-70, Dec. 1991.

[5] Q. Yang, C. Butler, and P. Baird, "Error compensation of touch trigger probes," Measurement: J. Int. Meas. Confed., vol. 18, no. 1, pp. 47-57, May 1996.

[6] E. J. Davis, W. J. Liao, G. F. Scott, and K. J. Stout, "Sources of measurement uncertainty when using coordinate measuring machines: A top-down systematic approach," in Proc. LAMDAMAP'95, Southampton, U.K., July 1995, pp. 185-201.

[7] W. Tyler Estler, S. D. Phillips, B. Borchardt, T. Hopp, C. Witzgall, M. Levenson, K. Eberhardt, M. McClain, Y. Shen, and X. Zhang, "Error compensation for CMM touch trigger probes," Precision Eng., vol. 19, no. 2-3, pp. 85-97, Oct./Nov. 1996.

[8] H. Haitjema, "Dynamic probe calibration in the micrometer region with nanometric accuracy," Precision Eng,, vol. 19, no. 2/3, pp. 98-104, Oct./Nov. 1996

[9] H. Pahk and J. Kim, "Application of microcomputer for assessing the probe lobing error and geometric errors of CMM's using commercial ring gauges," Int. J. Adv. Manufact. Technol., vol. 10, no. 3, pp. 208-218, 1995.

[10] L. Nawara and M. Kowalski, "The investigations on selected dynamical phenomena in the heads of multi-coordinate measuring devices," CIRP Ann., vol. 33, no. 1, pp. 373-375, 1984.

[11] R. P. Johnson, Q. Yang, and C. Butler, "Dynamic error characteristics of touch trigger probes fitted to coordinate measuring machines when scanning," unpublished.

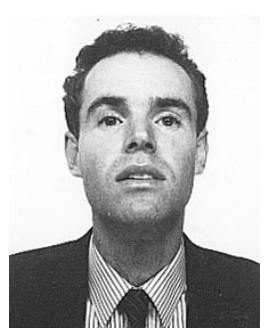

Roland P. Johnson was born in 1967. He received the degree in mechanical engineering from the Imperial College of Science and Technology, University of London, london, U.K., in 1988 and the Masters degree in industrial measurement systems in 1997.

Following this, he worked in industry including the technical development of bellows and the testing of electrical harnesses. At present, he is with the Brunel Centre for Manufacturing Metrology, Brunel University, Uxbridge, U.K. His current work includes the analysis of the error patterns of probes on coordinate measuring machines, particularly when scanning.

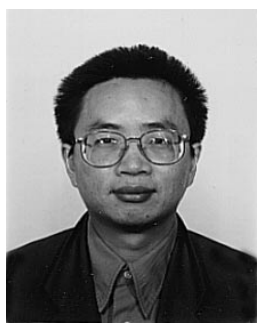

Qingping Yang (M'97) received the Diploma in instrumentation and measurement from Chengdu Aeronautical Polytechnic, Chengdu, China, in 1983 In 1988 he was awarded a scholarship to study at Brunel University, Uxbridge, U.K., and received the Ph.D. degree in 1992

In 1988 he was an Assistant Engineer and Deputy Head of the Department of Measurement and Testing in the Aircraft Structural Strength Research Institute (Ministry of Aerospace), Shaanxi Province, China. He is currently with Brunel University, Uxbridge, U.K. His current research interests include dimensional metrology, advanced sensors and transducers, virtual instrumentation, and measurement science. He has published more than 30 papers in these areas.

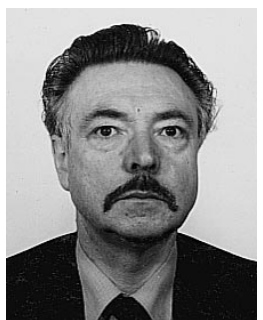

Clive Butler received the B.Sc. degree in physics from the University of Manchester, Manchester, U.K., in 1963, and the M.Sc. and Ph.D. degrees from Imperial College, London, U.K., in 1968.

He has held a number of industrial posts including Chief Physicist at OMT Ltd, and Director at Metronic Technology Ltd. and Digital Metrology Ltd. He was previously the Director of Research at Watford College of Technology, and is now a Reader at Brunel University, Uxbridge, U.K. His current research interests are in dimensional metrology and quality systems. He has presented more than 60 papers in applied optics, metrology, quality management, and printing technology.

Dr. Butler is a fellow of the Royal Society of Arts and a member of the Institute of Quality Assurance. 